\title{
Full-field measurement of micromotion around a cementless femoral stem using micro- CT imaging and radiopaque markers
}

V. Malfroy Camine ${ }^{1}$, H.A. Rüdiger ${ }^{2,3}$, D.P. Pioletti ${ }^{1}$ and A. Terrier ${ }^{1 *}$

1) Laboratory of Biomechanical Orthopedics, Ecole Polytechnique Fédérale de Lausanne, Lausanne, Switzerland

2)Service of Orthopedics and Traumatology, Lausanne University Hospital, Lausanne, Switzerland

3)Department of Orthopedic Surgery,Schulthess Clinic, Zürich, Switzerland

* Corresponding author:

AlexandreTerrier

Laboratory of Biomechanical Orthopedics

Ecole Polytechnique Fédérale de Lausanne

Station 19

1015 Lausanne

Switzerland

Tel: +41 216939498

Email: alexandre.terrier@epfl.ch

Article type: Original article

Word count: 3446

Keywords:Primary stability; Micromotion; Femoral stem; Micro-CT; Total Hip Replacement 


\begin{abstract}
A good primary stability of cementless femoral stems is essential for the long-term success of total hip arthroplasty.Experimental measurement of implant micromotion with linear variable differential transformers is commonly used to assess implant primary stability in pre-clinical testing. But these measurements are often limited to a few distinct points at the interface.New techniques based on micro-computed tomography (microCT) have recently been introduced, such as Digital Volume Correlation (DVC) or markers-based approaches. DVC is however limited to measurement around non-metallic implants due to metal-induced imaging artifacts, and markers-based techniques are confined to a small portion of the implant. In this paper, we present a technique based on micro-CT imaging and radiopaque markers to provide the first full-field micromotion measurement at the entire bone-implant interface of a cementless femoral stem implanted in a cadaveric femur.
\end{abstract}

Micromotion was measured during compression and torsion. Over 300 simultaneous measurement points were obtained. Micromotion amplitude ranged from 0 to $24 \mu \mathrm{m}$ in compression and from 0 to $49 \mu \mathrm{m}$ in torsion. Peakmicromotionwas distal in compression and proximal in torsion. The technique bias was $5.1 \mu \mathrm{m}$ and its repeatability standard deviation was $4 \mu \mathrm{m}$. The method was thus highly reliable and compared well with results obtained with linear variable differential transformers (LVDTs) reported in the literature.

These results indicate that this micro-CT based technique is perfectly relevant to observe local variations in primary stability around metallic implants. Possible applications include pre-clinical testing of implants and validation of patient-specific models for pre-operative planning. 


\section{Introduction}

2 During the past two decades, the number of cementless hip arthroplasties has increased 3 significantly from 13’650 procedures in 2003 to 27’031 in 2014 (Australian Orthopaedic

4 Association National Joint Replacement Registry, 2015), and it is now the preferred type of

5 fixation for patients under 70 years old.

6 However, the cumulative revision rate at 14 years reaches $8 \%$ and aseptic loosening remains

7 among the most common causes for revision of cementless femoral components. For this

8 reason, improving the long-term success of cementless femoral stems continues to be a major

9 focus in the field of total hip arthroplasty.

10 A good primary stability of the implant is widely recognized as the most important factor for

11 a successful cementless hip arthroplasty. Primary stability is characterized by the amount of

12 relative bone-implant micromotion at the interface, right after implantation and before 13 osseointegration takes place. Many researchers have reported that excessive implant

14 micromotion leads to fibrous tissue formation and failed bone ingrowth (Engh et al., 1992;

15 Pilliar et al., 1986; Søballe et al., 2009).

16 Much research in the recent years has focused on measuring bone-implant micromotion for

17 the pre-clinical testing of implants. An optimal experimental micromotion measurement

18 technique for the pre-clinical testing of femoral stems should be able to evaluate micromotion

19 at every point of the bone-implant interface while having a bias below $10 \mu \mathrm{m}$ (Viceconti et

20 al., 2000). Considering the maximum micromotion still allowing osseointegration is around

$21100 \mu \mathrm{m}$, this bias value would represent a relative error of $10 \%$.Current techniques available

22 to measure implant micromotion rely mostly on linear variable differential transformers

23 (LVDTs) (Enoksen et al., 2014; Fottner et al., 2009; Kassi et al., 2005; Monti et al., 1999;

24 Pettersen et al., 2009; Østbyhaug et al., 2010). Despite their excellent accuracy, they allow 
25 only a handful of simultaneous measurement points. Finite element (FE) modelling is another 26 popular method to estimate micromotion of cementless stems. It provides information on 27 local micromotion and can be used for the pre-clinical testing of implants (Abdul-Kadir et al., 28 2008; Bah et al., 2015; van der Ploeg et al., 2011; Viceconti et al., 2006) as well as for 29 patient-specific pre-operative planning(Pettersen et al., 2009; Reggiani et al., 2007). But 30 experimental validation of FE models predictions remains challenging,restraining a more 31 extensive use of these models in clinical practice (Taylor and Prendergast, 2015).More

32 recently, micromotion measurement techniques based on micro-computed tomography 33 (micro-CT) imaging were introduced, and demonstrated great potential. Notwithstanding the 34 very high number of measurement points they can collect, they were limited to measurement 35 around non-metallic implants due to imaging artifacts(Sukjamsri et al., 2015) or confined to a 36 small portion of the implant (Gortchacow et al., 2012; 2011).

37 In the presentstudy, we extend a micromotion measurement technique based on radiopaque 38 markers and micro-CT imaging (Gortchacow et al., 2011)to measure three 39 dimensionalmicromotion at the entirebone-implant interface of a cementless femoral stem 40 implanted in a cadaveric femur. The method will allow to measure micromotion for axial 41 compression and torsion. Our objective is to guarantee abias inferior to $10 \mu \mathrm{m}$ and a 42 goodrepeatability to enable rigorous pre-clinical testing of cementless implants primary 43 stability. 


\subsection{Cadaveric femur and femoral stem preparation}

46 A left human cadaveric femur, formalin-fixed, was prepared for implantation by a senior

47 orthopedic surgeon. The surgeon performed femoral neck osteotomy and femoral broaching

48 according to the recommendations of the implant's manufacturer. After broaching, around

491000 stainless steel spherical markers of diameter $600 \mu \mathrm{m}$ (MPS Micro Precision Systems

50 AG, Biel, Switzerland) were manually press-fitted on the endosteal surface of the femoral

51 canal and the cancellous bone of the metaphysis using a spatula. Appropriate care was taken

52 to get a uniform distribution of bone markers in the canal (Fig.1).

53 A collared, straight cementless femoral stem with a standard offset neck (Corail® Hip

54 System, size 11, DePuy Synthes Joint Reconstruction, Warsaw, IN, USA) was selected for

55 implantation. The stem is made of forged titanium alloy (TiAl6V4) and is fully coated with

$56155 \mu \mathrm{m}$ of hydroxyapatite. To facilitate the accommodation of the bone-implant construct

57 inside the experimental setup, the stem femoral neck was cut $27 \mathrm{~mm}$ medial and parallel to

58 the implant extraction threaded hole axis. 30 tantalum spherical markers of diameter $800 \mu \mathrm{m}$

59 (X-medics Scandinavia, Frederiksberg, Denmark) were glued (Loctite 401, Loctite

60 Corporation, Dublin, Ireland) on the stem surface, within drilled holes of $1 \mathrm{~mm}$ depth and 850

$61 \mu \mathrm{m}$ diameter(Fig.1). The surgeon then proceeded to the femoral stem insertion in the

62 broached femur.

\subsection{Loading devices}

64 Two custom loading devices were developed to apply axial compression and torsion on the

65 stem. The loading devices had to fit inside a micro-CT scanner and had to be sufficiently 66 permeable to X-ray. Each device was composed of two parts: the loading system and the 67 sample holder, enclosed in a $2 \mathrm{~mm}$ thick tube made of 6060 aluminum alloy (Fig.1). 
68 The compression device was modified from an existing one (Gortchacow et al., 2012). The 69 distal part of the femur was cut away at approximately $220 \mathrm{~mm}$ from the tip of the greater

70 trochanter. A template was used to pot the distal femur and ensure its alignment (load axis

71 along stem axis) inside the device, using the stem extractionthreaded hole. The femur was

72 distally potted with epoxy resin (Neukadur Multicast 20, Altropol Kunstoff GmbH,

73 Stockelsdorf, Germany), $30 \mathrm{~mm}$ away from the distal end of the stem.Minimal reaming of the

74 surface of the greater trochanter laterally (2-3 mm)was performed to enable proper fitting

75 inside the device. The applied load was monitored by a load cell (LCM202-5KN, Omega

76 Engineering, Inc., Stamford, CO, USA).

77 The torsion device applied an axial torsion on the bone-stem system. The proximal part of the

78 stem was restrained by a clamping system. The stem extraction threaded hole was used to 79 ensure stem alignment along the torsion axis. The stem neck was clamped by two steel cone 80 point screws. The distal femur and the proximal clamping system were potted with epoxy 81 resin inside a template, before insertion in the device. A torsion was applied to the distal 82 femur through a rotary shaft driven by a worm gear. The torque was monitored by a reaction 83 torque cell (TQM301-45N, Omega Engineering, Inc., Stamford, CO, USA).

$84 \quad$ 2.3. Micro-CT scanning protocol

85 To measure micromotion, the bone-implant interface was first scanned during loading and 86 then after loading with a micro-CT scanner (Skyscan 1076 in vivo micro-CT, Bruker micro87 CT, Kontich, Belgium). These two scans are referred to hereafter as loaded scan and 88 unloaded scan respectively. The acquisition parameters for the scans were the following: $891 \mathrm{~mm}$ aluminum filter, voltage $100 \mathrm{kV}$, current $100 \mu \mathrm{A}$, exposure time $310 \mathrm{~ms}$, rotation step $90 \quad 0.7^{\circ}, 360^{\circ}$ scanning, scanning width $68 \mathrm{~mm}$, and frame averaging 2 . The scanning length was $9121 \mathrm{~mm}$. To cover the whole implant length, 7 scans at different positions along the stem were 
92 combined by moving the motorized sample's stage accordingly. Scanning duration for one 21

$93 \mathrm{~mm}$ scan was $24 \mathrm{~min}$, resulting in $170 \mathrm{~min}$ of scanning to cover the whole stem. Scans were

94 then reconstructed to a final isotropic voxel size of $35 \mu \mathrm{m}$ (NRecon v 1.6.10.4, Bruker micro-

95 CT, Kontich, Belgium). A ring artifact correction of level 4 and a beam hardening correction

96 of $20 \%$ were applied to improve the image quality.

\section{2.4. Image processing and micromotion computation}

98 The reconstructed images were processed in Amira (Amira v6.0.1, FEI, Hillsboro, OR, 99 USA). Segmentation of bone and implant markers was completely automatized, using the 100 difference in size and radiopacity of bone and implant markers (Fig.2). The centroids of all 101 markers were extracted and filtered by size to eliminate noise and clusters of contiguous 102 markers.

103 Micromotion analysis was performed by a custom algorithm (Matlab r2016a, The 104 Mathworks, Inc., Natick, MA, USA). The loaded and unloaded scans did not share the same 105 coordinate system. The coordinate system of the unloaded scan was used as a reference. The 106 implant was considered rigid so that the coordinate systems of both scans could be aligned 107 using rigid body registration. The correspondence between implant markers in the loaded and 108 unloaded scans was found using an iterative closest point (ICP) algorithm (Besl and McKay, 109 1992). The rigid transformation matrix between the loaded and unloaded scans was then 110 computed, and applied to all markers from the loaded scan, so that in the end, all markers 111 from the loaded and unloaded scans were in the same coordinate system.

112 Micromotion was defined as the three dimensional displacement between corresponding 113 loaded and unloaded bone markers. The correspondence between bone markers was 114 computed with the ICP algorithm. Mismatched markers were then eliminated using median 115 absolute deviation to remove outliers (Leys et al., 2013). The micromotion vector was 
116 separated into components tangential and normal to the stem surface. Micromotion was then

117 interpolated using natural neighbor interpolation and displayed on the stem surface.

\section{$118 \quad$ 2.5. Micromotion measurement in compression and torsion}

119 For compression testing, a load of $1800 \mathrm{~N}$ was applied on the stem. The load was chosen

120 according to the average load during walking measured with instrumented hip implants

121 (Bergmann et al., 2010a; 2010b). The bone was preconditioned with 50 compressive load

122 cycles before compression testing. For torsion testing, a torque of $17 \mathrm{~N} \mathrm{~m}$ was applied on the

123 stem. Moment and direction were chosen according to average moment acting on

124 instrumented hip implants during stair climbing (Bergmann et al., 2010b; 2010a). The bone

125 was preconditioned with 50 torsional load cycles before torsion testing.All tests were 126 performed at room temperature.

127 2.6. Bias and repeatability estimation

128 Bias and repeatability were measured in both compression and torsion to evaluate the 129 technique reliability. The bias (a measure of the differencebetween the average of 130 measurements made on the same object and its true value) was estimated by measuring 131 micromotion between three pairs of successive unloaded scans (Fig. 3). Each 3D component 132 of micromotion followed a normal distribution, with mean 0 . The bias was defined as the $13395 \%$ confidence interval (95\% CI) of micromotion measurement, corresponding to $134 \pm 1.96 * \mathrm{SD}$, where SD is the standard deviation of micromotion pooled over the three pairs of 135 measurement.

136 To estimate repeatability, micromotion measurements in compression and torsion were 137 repeated three times (Fig. 3), under repeatability conditions (same laboratory, same operator, 138 same apparatus, and all tests performed on the same day). Corresponding markers were 
139 matched between the three pairs of measurements. The repeatability standard deviation (sr)

140 was calculated as the pooled standard deviation of repeated measurements. The 95\%

141 repeatability limit (r) (the maximum difference between two results obtained under

142 repeatability conditions that can be attributed to the test method precision) was defined as

$1431.96 * \sqrt{ } 2 *$ sr according to current ASTM recommendations (ASTM, 2013).

$144 \quad$ 2.7. Data analysis and statistics

145 For measurement analysis, the femoral stem was divided into three zones: the metaphyseal 146 zone, the middle diaphyseal zone, and the distal diaphyseal zone, similar to the 147 recommendations of Gruen et al. (1979). Normal and tangential micromotion in compression 148 and torsion were compared in each zonewith a Mann-Whitney U test. For each loading case, 149 micromotion between zones were also compared using the same Mann-Whitney U test. 


\section{Results}

$151 \quad 3.1$. Bias and repeatability

152 Micromotion was simultaneously measured at 313 points on the bone-implant interface for 153 compression and 337 points for torsion. The bias of the method reached a maximum of 5.1 $154 \mu \mathrm{m}$ (Table 1). The bias was consistent between directions as well as between loading cases. 155 The repeatability standard deviation (sr) ranged from $3.1 \mu \mathrm{m}$ to $4.1 \mu \mathrm{m}$. It was also 156 comparable between directions and loading cases. The repeatability limitreached a maximum 157 of $10.6 \mu \mathrm{m}$ for compression and $11.5 \mu \mathrm{m}$ for torsion.

\subsection{Micromotion in compression and torsion}

159 In compression, normal micromotion wasbelow $6 \mu \mathrm{m}$ around 95\% of the stem surface but

160 reached $24 \mu \mathrm{m}$ atthe tip of the stem (Fig. 4\& Movie 1). Tangential micromotion was higher 161 than normal micromotion and concentrated on the stem's middle and distaldiaphyseal zones. 162 In torsion, high micromotion was concentrated on the stem's metaphyseal and middle 163 diaphyseal parts (Fig 4 \& Movie 2).

164 Median micromotion was higher in torsion than in compression (Table 2).In compression, 165 micromotion was low proximally and higher distally, whereas in torsion micromotion was 166 high proximally and lower distally. For both loading cases, the differences between 167 micromotion distribution in each zone of the stem was significant. Absolute micromotion was 168 significantly $(\mathrm{p}<0.0001)$ higher in torsion than in compression in the metaphyseal and middle 169 diaphyseal zones, while it was significantly lower on the distal diaphysis (Fig. 5). 


\section{Discussion}

171 Micro-CT based techniques such as Digital Volume Correlation (DVC) have been recently

172 used to measure displacement and strain fields in the bone (Roberts et al., 2014). However,

173 extension of micro-CT DVC to measurements at the bone-implant interface faces

174 complications due to artifacts generated by thick metal implants: in consequence of the high

175 atomic number of the metallic implant, the bone would be obscured, streak artefacts would be

176 generated and beam hardening would impact the gray levels at the bone-implant interface

177 (Boas and Fleischmann, 2012). Our aim was to develop a new technique to measure

178 micromotion all around the femur-stem interface, with a bias lower than $10 \mu \mathrm{m}$ and a good

179 repeatability to allow thorough pre-clinical testing of implants. We proposed a methodology

180 based on radiopaque markers and micro-CT imaging, and measured micromotion around a

181 cementless stem in a cadaveric femur under compressive and torsional loadings. Instead of

182 imaging directly the interface, the radiopaque markers representing the bone and implant

183 surfaces were used. In combination with appropriate scanning parameters, this approach

184 represents the first reported experimental technique leading to a full-field map of interface

185 micromotion around the entire stem.

186 We tested and compared micromotion values in compression and torsion. We obtained over

187300 measurement points spread at the bone-stem interface, and were able to observe local

188 variations of micromotion depending on the loading case. The maximum bias was $5.1 \mu \mathrm{m}$

189 and the repeatability limit was $11.5 \mu \mathrm{m}$, which demonstrates that the technique ishighly

190 reliable. The direction of micromotion was distinctly inferior for compression, which is

191 consistent with the axial compressive loading applied. In torsion, normal and tangential

192 micromotion were both comparable in amplitude, in good agreement with an axial torsion of

193 the stem. The stem used in this study is designed to achieve metaphyseal fixation, and

194 consistently, bone-implant gap was particularly low in this zone (Fig S1\& Movie 3). The 
195 metaphyseal region corresponded indeed to a region of low micromotion in compression, but 196 we observed high micromotion in torsion. Generally speaking, there didn’t seem to be a 197 direct visual correspondence between local bone-implant gap and micromotion.

198 The validity of the rigid body assumption for the implant has been rigorously verified by 199 calculating the root mean square error (RMSE) of the rigid body registration. The RMSE was 200 approximately $3 \mu \mathrm{m}$, negligible compared to the expected values of interfacial micromotion. 201 Scanning duration was 170 min which is a rather long scanning time. It remained however 202 acceptable, because load relaxation was limited to $1 \%$ load loss in compression and $4 \%$ in 203 torsion during this time.The fixation of the bone markers was challenging. The markers 204 diameter was chosen to let them penetrate the bone trabeculae, but in the distal medullary 205 canal, cancellous bone is rare. In this region, the markers were simply deposited on the 206 endosteal surface of the bone. Bone markers contiguous to the stem and not well fixed to the 207 bone were a major concern because they could move along with the implant and lead to the 208 underestimation of micromotion. To avoid this issue, the automatic segmentation script 209 removed all bone markers that were in contact with the femoral stem from the measurement.

210 Despite all our efforts, some markers in the metaphyseal area did not enter bone trabeculae 211 and were in direct contact with both the bone and the implant. This situation modifies the 212 original interface and can have an impact on the measurement. However, our results were 213 compatible with measurements obtained with LVDTs. This encourages us to think that this 214 modification of the interface does not change dramatically the magnitude of micromotion. 215 With our method, the distal femur was cemented at approximately half the length of the 216 femur (i.e. at the level of the isthmus) for both loading cases, which is not representative of 217 the actual constraints on the bone and modifies the stress and strain distributions in the femur. 218 However, we were limited by the size of our micro-CT scanner and moving the constraint 219 further away was impossible. For the same reason, the compressive loading was applied on 
the stem extraction threaded hole of the stem shoulder instead of the implant neck and axial

221 compression and torsion were tested separately, which does not represent a physiological

222 loading of the stem. Finally, this study was limited to one formalin-fixed femur, for which the

223 mechanical properties are degraded compared to a fresh bone (Currey et al., 1995; Öhman et

224 al., 2008; Stefan et al., 2010). Nevertheless, this allowed us to demonstrate the feasibility of

225 the technique, while avoiding tissue degradation which would have emerged with a fresh

226 frozen bone during the multiple tests conducted in this study.

227 In general, our results were in good agreement with results reported in the literature. We 228 measured absolute micromotion values that ranged from 0 to $40 \mu \mathrm{m}$. Pettersen et al. 229 (2009)measured micromotion in the same range using LVDTs around straight cementless 230 stems in fresh-frozen femurs. Similarly, Abdul-Kadir et al. (2008)measured micromotion of 231 up to $20 \mu \mathrm{m}$ with LVDTs for an axial compression on the stem shoulder, which is identical to 232 our results. We found higher micromotion in torsion (stair climbing) than in compression 233 (walking). This result compares well with results from Enoksen et al. (2014)and Kassi et al. 234 (2005)obtained with LVDTs or with measurements from postmortem retrieval sections by 235 Mann et al. (2012). The patterns of micromotion revealed that for axial compression, 236 micromotion was low proximally and high distally. Pancanti et al. (2003)observed a similar 237 pattern with a FE model. Moreover, this finding is consistent with the femoral stem design, 238 thought to achieve stabilization in the metaphyseal area (Vidalain, 2010). In torsion, we 239 found high micromotion proximally and lower micromotion distally. Kassi et al. (2005)and 240 Pancanti et al. (2003)also measured higher micromotion proximally but they had a second 241 region of high micromotion at the tip of the stem. Differences in loading and constraints can 242 be possible explanations for this variation.

243 The reliability of the method was evaluated through bias and repeatability. Maximum bias 244 was $5.1 \mu \mathrm{m}$. Although this value is high compared to the accuracy that can be obtained with 
245 LVDTs, it remains sufficient to be used for the validation of FE models or for comparing

246 different stems designs. The repeatability standard deviation reached a maximum of $4.1 \mu \mathrm{m}$.

247 This value encompasses random errors due to the precision of loading, to the transmission of

248 load to the femoral stem, and to the viscoelastic behavior of bone. It can be compared to

249 similar measures of repeatability performed for LVDTs setups: Monti et al. (1999)obtained a

250 maximum value of $5 \mu \mathrm{m}$ for intra-specimen standard deviation, Viceconti et al.

251 (2000)measured a maximum intra-specimen variability of $9 \mu \mathrm{m}$, while Kassi et al. (2005)and

252 Østbyhaug et al. (2010)got values of $3 \mu \mathrm{m}$ and $1.65 \mu \mathrm{m}$ respectively for repeatability

253 standard deviation. The repeatability of micro-CT based micromotion measurement is thus

254 similar to the repeatability of LVDT-based micromotion measurement.

255 The technique we proposed here relies on radiopaque markers with different radiopacity and

256 size attached to the bone and the implant to overcome the difficulty of imaging directly the

257 bone-implant interface. The bias and repeatability of the technique were comparable to those

258 of LVDT-based measurements, making it a technique as reliable as the current gold standard.

259 This resulted in a unique full-field map of micromotion around a cementless femoral stem,

260 that may be used to compare the local effects of different implant designs or to corroborate

261 FE results. Notably, the validation of patient-specific models that predict the level of bone-

262 implant micromotion may be a promising application of the proposed technique. Indeed, a

263 validated model could be used for pre-operative planning to compare the performance of

264 different stem designs, of different surgical techniques, or of different stem positions for a

265 given patient. This may improve our understanding of primary implant stability and may lead

266 to enhanced long-term success of cementless total hip arthroplasty. 


\section{Acknowledgements}

268 This work was supported by the Swiss National Science Foundation (\#141152) and the 269 SwissLife Jubiläumstiftung. The authors would like to thank DePuy Synthes (DePuy Synthes

270 Joint Reconstruction, Warsaw, IN, USA)for donating the femoral stem and providing CAD

271 files, the Lausanne University Hospital (CHUV) for providing the cadaveric femur, and

272 Alejandro Dominguez and the Centre Universitaire Romand de Médecine Légale (CURML)

273 for the CT-scans of the femur.

274 


\section{References}

276 Abdul-Kadir, M.R., Hansen, U., Klabunde, R., Lucas, D., Amis, A., 2008. Finite element modelling of primary hip stem stability: The effect of interference fit. Journal of Biomechanics 41, 587-594.

ASTM, 2013. Standard practice for use of the terms precision and bias in ASTM test methods. In E177 ed: Subcommittee E11.20 on test method evaluation and quality control. West Conshohocken: ASTM International.

Australian Orthopaedic Association National Joint Replacement Registry, 2015. Annual Report. Adelaide:AOA.

Bah, M.T., Shi, J., Heller, M.O., Suchier, Y., Lefebvre, F., Young, P., King, L., Dunlop, D.G., Boettcher, M., Draper, E., Browne, M., 2015. Inter-subject variability effects on the primary stability of a short cementless femoral stem. Journal of Biomechanics 48, 1032-1042.

Bergmann, G., Graichen, F., Rohlmann, A., Bender, A., Heinlein, B., Duda, G.N., Heller, M.O., Morlock, M.M., 2010a. Realistic loads for testing hip implants. Bio-Medical Materials and Engineering 20, 65-75.

Bergmann, G., Graichen, F., Rohlmann, A., Bender, A., Heinlein, B., Duda, G.N., Heller, M.O., Morlock, M.M., 2010b. Erratum: Realistic loads for testing hip implants (BioMedical Materials and Engineering (2010) 20 (65-75)). Bio-Medical Materials and Engineering 20, 381.

Besl, P.J., McKay, N.D., 1992. A Method for Registration of 3-D Shapes. IEEE Transactions on Pattern Analysis and Machine Intelligence 14, 239-256.

297 Boas, F.E., Fleischmann, D., 2012. CT artifacts: Causes and reduction techniques. Imaging in Medicine 4, 229-240.

299 Currey, J.D., Brear, K., Zioupos, P., Reilly, G.C., 1995. Effect of formaldehyde fixation on 
some mechanical properties of bovine bone. Biomaterials 16, 1267-1271.

301 Engh, C.A., O'Connor, D., Jasty, M., McGovern, T.F., Bobyn, J.D., Harris, W.H., 1992. Quantification of Implant Micromotion, Strain Shielding, and Bone Resorption With Porous-Coated Anatomic Medullary Locking Femoral Prostheses. Clinical Orthopaedics and Related Research 285, 13-29.

Enoksen, C.H., Gjerdet, N.R., Klaksvik, J., Arthursson, A.J., Schnell-Husby, O., Wik, T.S., 2014. Initial stability of an uncemented femoral stem with modular necks. An experimental study in human cadaver femurs. Clinical Biomechanics 29, 330-335.

Fottner, A., Schmid, M., Birkenmaier, C., Mazoochian, F., Plitz, W., Jansson, V., 2009. Biomechanical evaluation of two types of short-stemmed hip prostheses compared to the trust plate prosthesis by three-dimensional measurement of micromotions. Clinical Biomechanics 24, 429-434.

Gortchacow, M., Wettstein, M., Pioletti, D.P., Müller-Gerbl, M., Terrier, A., 2012. Simultaneous and multisite measure of micromotion, subsidence and gap to evaluate femoral stem stability. Journal of Biomechanics 45, 1232-1238.

Gortchacow, M., Wettstein, M., Pioletti, D.P., Terrier, A., 2011. A new technique to measure

Gruen, T.A., McNeice, G.M., Amstutz, H.C., 1979. "Modes of failure” of cemented stemtype femoral components: a radiographic analysis of loosening. Clinical Orthopaedics and Related Research 17-27.

321 Kassi, J.-P., Heller, M.O., Stoeckle, U., Perka, C., Duda, G.N., 2005. Stair climbing is more critical than walking in pre-clinical assessment of primary stability in cementless THA in vitro. Journal of Biomechanics 38, 1143-1154.

324 Leys, C., Ley, C., Klein, O., Bernard, P., Licata, L., 2013. Detecting outliers: Do not use 
standard deviation around the mean, use absolute deviation around the median. Journal of Experimental Social Psychology 49, 764-766.

Mann, K.A., Miller, M.A., Costa, P.A., Race, A., Izant, T.H., 2012. Interface Micromotion of Uncemented Femoral Components from Postmortem Retrieved Total Hip Replacements. The Journal of arthroplasty 27, 238-245.

Monti, L., Cristofolini, L., Viceconti, M., 1999. Methods for Quantitative Analysis of the Primary Stability in Uncemented Hip Prostheses. Artificial Organs 23, 851-859.

Öhman, C., Dall’Ara, E., Baleani, M., Jan, S.V.S., Viceconti, M., 2008. The effects of embalming using a $4 \%$ formalin solution on the compressive mechanical properties of human cortical bone. Clinical Biomechanics 23, 1294-1298.

Pancanti, A., Bernakiewicz, M., Viceconti, M., 2003. The primary stability of a cementless stem varies between subjects as much as between activities. Journal of Biomechanics 36, 777-785.

Pettersen, S.H., Wik, T.S., Skallerud, B., 2009. Subject specific finite element analysis of implant stability for a cementless femoral stem. Clinical Biomechanics 24, 480-487.

Pilliar, R.M., Lee, J.M., Maniatopoulos, C., 1986. Observations on the Effect of Movement on Bone Ingrowth into Porous-Surfaced Implants. Clinical Orthopaedics and Related Research 208, 108-113.

Reggiani, B., Cristofolini, L., Varini, E., Viceconti, M., 2007. Predicting the subject-specific primary stability of cementless implants during pre-operative planning: Preliminary

347 Roberts, B.C., Perilli, E., Reynolds, K.J., 2014. Application of the digital volume correlation technique for the measurement of displacement and strain fields in bone: A literature review. Journal of Biomechanics 47, 923-934. 
Stefan, U., Michael, B., Werner, S., 2010. Effects of three different preservation methods on the mechanical properties of human and bovine cortical bone. Bone 47, 1048-1053. Emery, R., Hansen, U., 2015. Digital volume correlation and micro-CT: An in-vitro technique for measuring full-field interface micromotion around polyethylene implants. Journal of Biomechanics 48, 3447-3454.

Søballe, K., Brockstedt-Rasmussen, H., Hansen, E.S., Bünger, C., 2009. Hydroxyapatite coating modifies implant membrane formation. Acta Orthopaedica Scandinavica 63, $128-140$.

Taylor, M., Prendergast, P.J., 2015. Four decades of finite element analysis of orthopaedic Toward a more realistic prediction of peri-prosthetic micromotions. Journal of Orthopaedic Research 30, 1147-1154.

Viceconti, M., Brusi, G., Pancanti, A., Cristofolini, L., 2006. Primary stability of an anatomical cementless hip stem: A statistical analysis. Journal of Biomechanics 39, 1169-1179.

Viceconti, M., Muccini, R., Bernakiewicz, M., Baleani, M., Cristofolini, L., 2000. Largesliding contact elements accurately predict levels of bone-implant micromotion relevant to osseointegration. Journal of Biomechanics 33, 1611-1618.

Vidalain, J.P., 2010. Twenty-year results of the cementless Corail stem. International Orthopaedics 35, 189-194. 
377 Figure 1: Experimental setup. (a) Bone markers spread inside the femoral canal (left). 378 Stem neck cut and implant markers stuck on implant surface (right). (b) Compression loading device. The distal femur is cemented (black). Compression is applied through a cylinder (yellow) driven by a screw jack (green) and is controlled by a load cell (blue) (c) 381 Torsion loading device. The proximal stem is restrained by a clamping system (yellow).

382 The proximal stem and the distal femur are cemented (black). Torsion is applied through a worm gear (green) and is controlled by a torque sensor (blue).

Figure 2: Image processing and micromotion computation. Bone and implant markers are segmented on micro-CT scans. Implant markers from the loaded scan are superimposed to implant markers from the unloaded scan. Micromotion is the displacement between corresponding bone markers from the registered loaded scan to the unloaded scan.

Figure 3: Bias and repeatability estimation protocols. Both protocols were applied successively for compression and torsion (a) Three pairs of unloaded scans $(U)$ are performed. For each pair of scan (Rep), micromotion is measured. Bias is estimated on these three repeated measurements. (b) Three pairs of unloaded $(U)$ and loaded (L) scans are performed. For each pair of scan (Rep), micromotion is measured.

394 Repeatability is estimated on these three repeated measurements.

395 Figure 4: Normal, tangential, and absolute micromotion measured around a cementless femoral

396 stem - Anterior/lateral and posterior/medial views of the stem displayed successively from left to

397 right for each case. Top row shows results obtained in compression. The bottom row shows

398 results obtained in torsion.

399 Figure 5: Distribution of normal, tangential, and absolute micromotion in compression and

400 torsion by zone of the femoral stem. Box plots show median value (white line), $1^{\text {st }}$ and $3^{\text {rd }}$

401 quartiles (bottom and top of the box), and minimum and maximum values (whiskers). Stars $(*$ )

402 indicate significant difference between pairs of distributions ( $p$-value $<0.0001)$. 


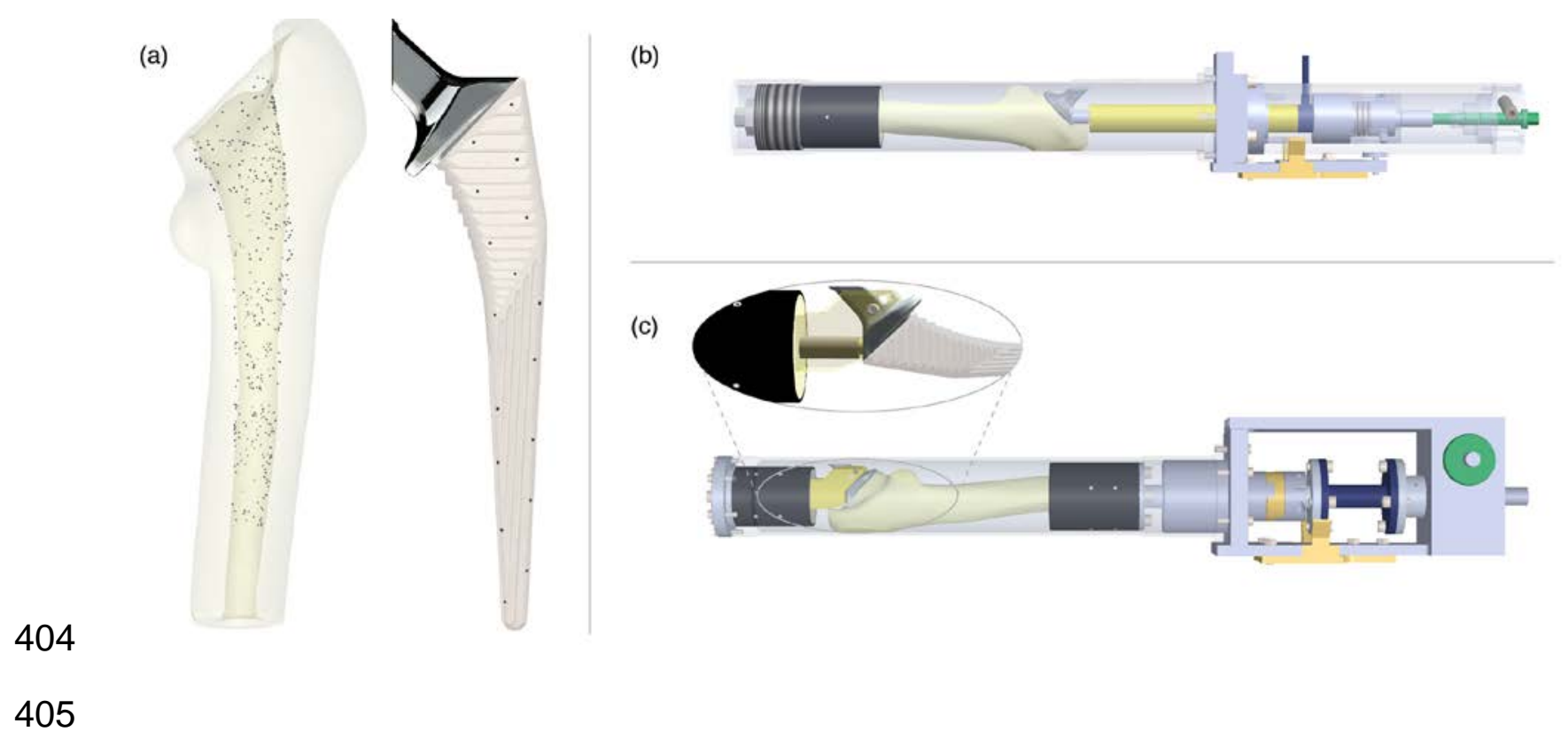




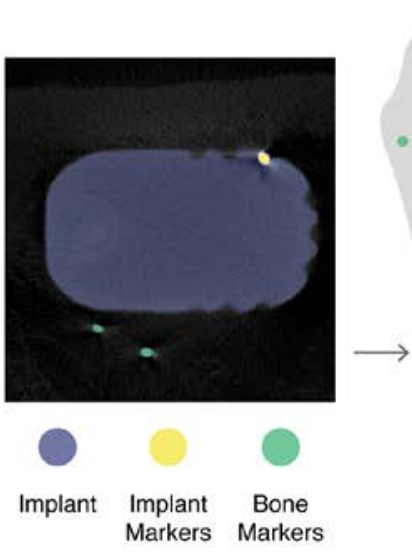

Segmentation

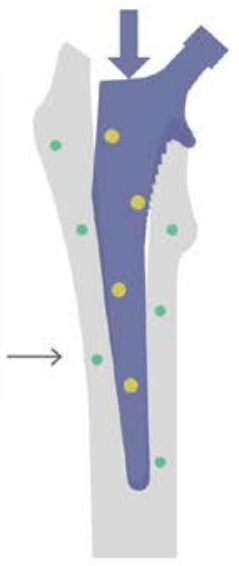

Loaded scan

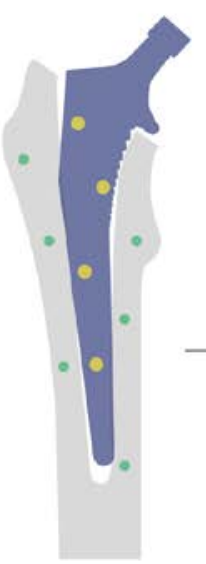

Unloaded scan
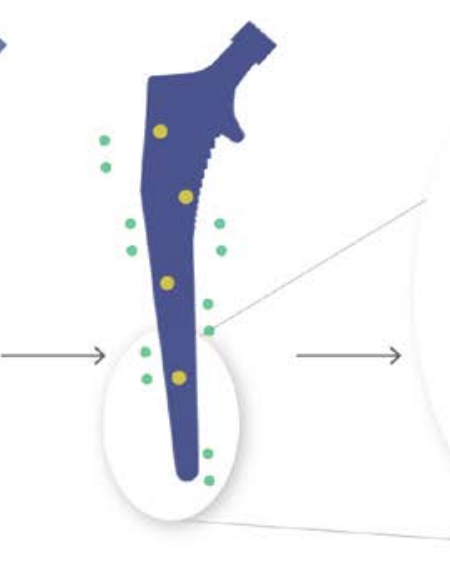

Rigid body registration

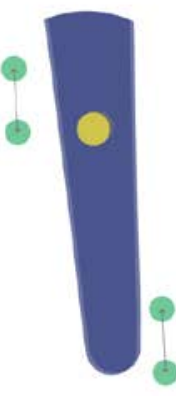

Micromotion computation 
(a)

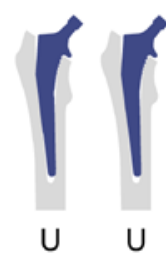

Rep \#1

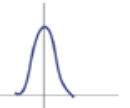

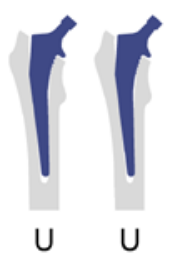

Rep \#2
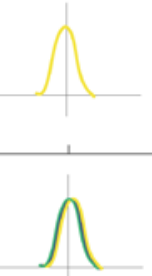

408

Bias (b)
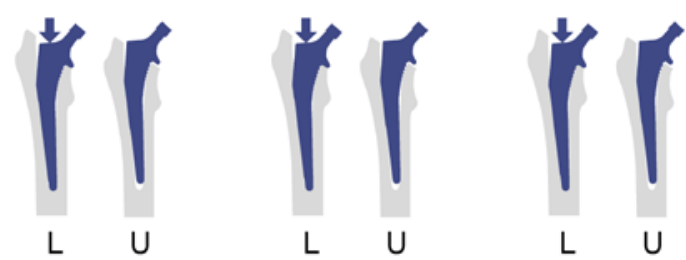

Rep \#1

Rep \#2

Rep \#3

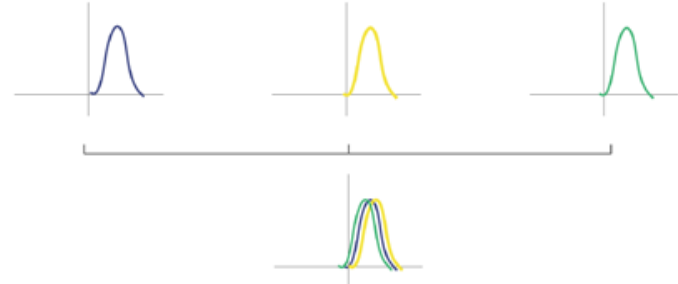

Repeatability

409 

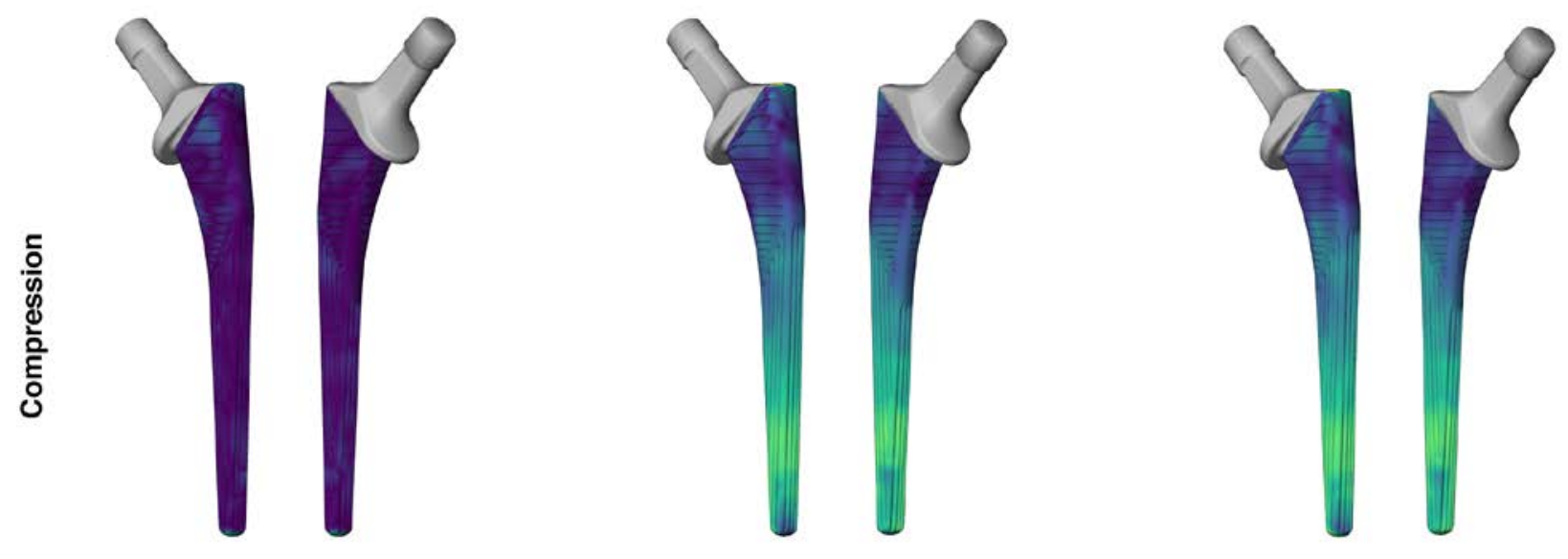

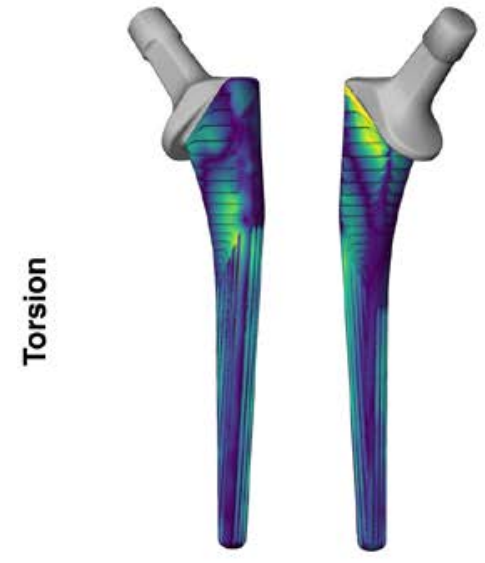

Normal Micromotion

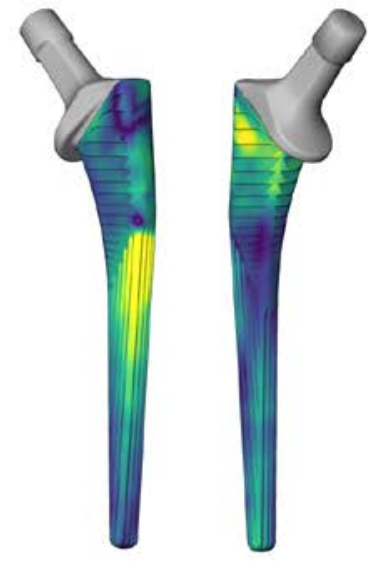

Tangential Micromotion

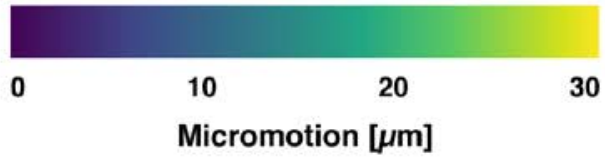

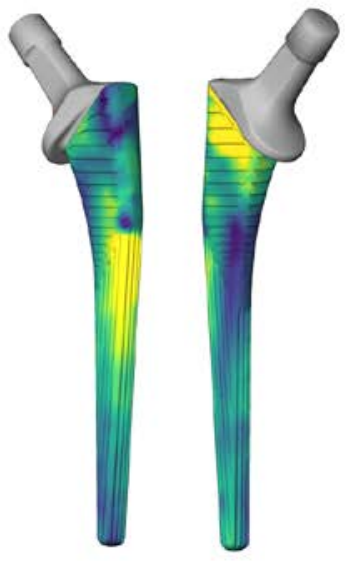

Absolute Micromotion 


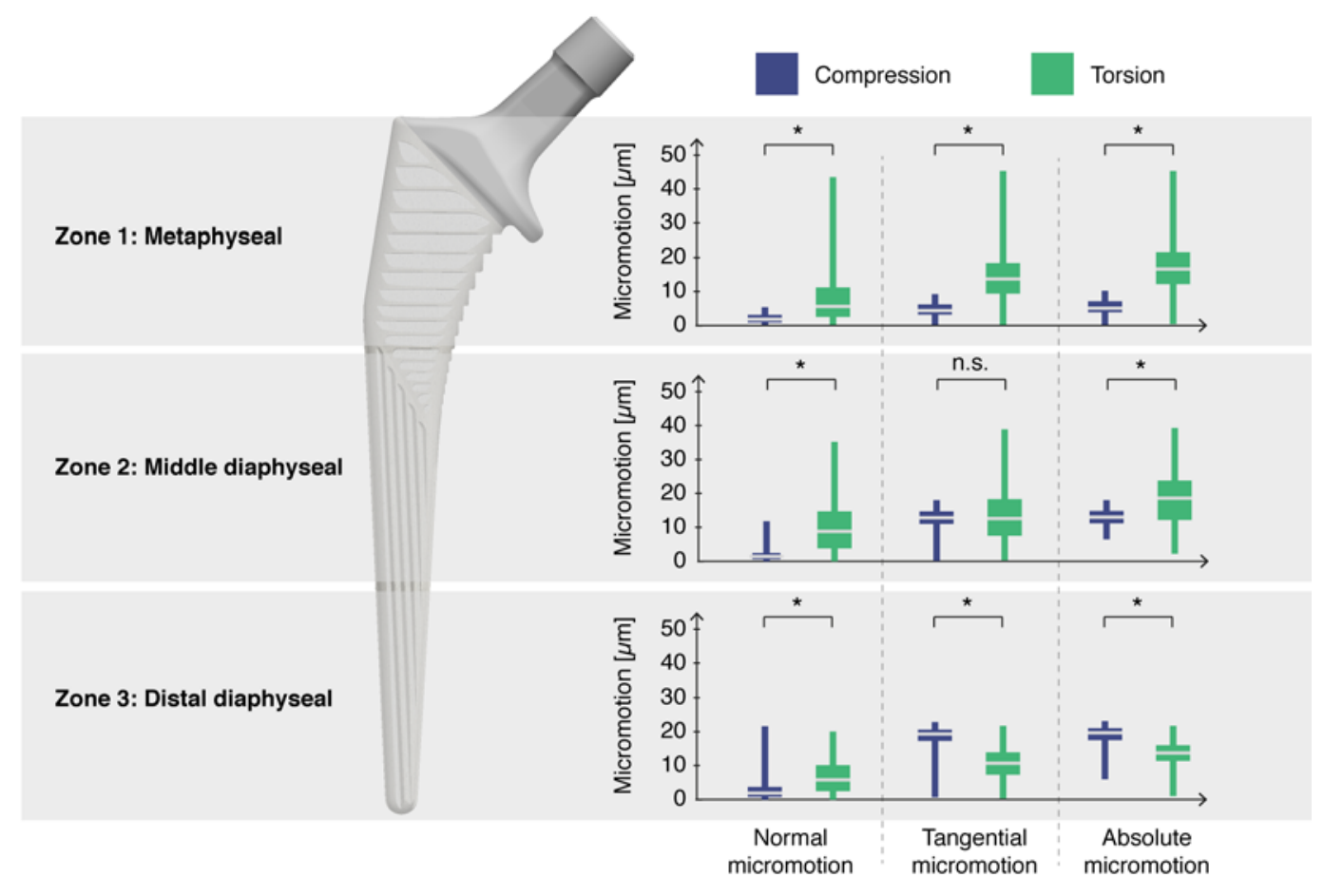

413 
414 Table 1:Reliability assessment of Micro-CT based measurement of micromotion - Values

415 expressed in micrometers. SD: bias standard deviation; 95\% CI: bias 95\% confidence interval;

416 sr: repeatability standard deviation; r: repeatability 95\% limit.

\begin{tabular}{lllll}
\hline & Bias & & \multicolumn{2}{l}{ Repeatability } \\
\cline { 2 - 5 } & SD & $95 \%$ CI & sr & r \\
\hline Compression & & & & \\
Lateral to medial & 2.4 & 4.7 & 3.2 & 9.0 \\
Anterior to posterior & 2.6 & 5.1 & 3.8 & 10.6 \\
Inferior to superior & 1.9 & 3.7 & 3.1 & 8.7 \\
Torsion $\quad$ & & & & \\
Lateral to medial & 2.4 & 4.7 & 3.9 & 10.9 \\
Anterior to posterior & 2.4 & 4.7 & 4.0 & 11.2 \\
Inferior to superior & 1.9 & 3.7 & 4.1 & 11.5 \\
\hline
\end{tabular}

417

418 
419 Table 2: Minimum, maximum, and median micromotion $(\mu \mathrm{m})$ along the different anatomical axis

420 for compression and torsion.

\begin{tabular}{llll}
\hline & Min & Max & Median \\
\hline Compression & & & \\
Lateral to medial & -7.8 & 10.8 & 1.9 \\
Anterior to Posterior & -12 & 13.7 & 0.4 \\
Inferior to Superior & -24 & 5.0 & 12.3 \\
Absolute & 0.8 & 24 & 13.3 \\
& & & \\
Torsion $\quad-32.5$ & 44.1 & -1.5 \\
Lateral to medial & -42.2 & 33.8 & -7.5 \\
Anterior to Posterior & -7.8 & 15.2 & 0.8 \\
Inferior to Superior & & & \\
Absolute & 2.4 & 48.7 & 20.9 \\
\hline
\end{tabular}

421

422 